\title{
Detection of Capsular Polysaccharide in Serum for the Diagnosis of Pneumococcal Pneumonia: Clinical and Experimental Evaluation
}

\author{
Andreas Schaffiner, Claudia Michel-Harder, \\ and Sibel Yeginsoy
}

Department of Medicine, University of Zurich Medical School, Switzerland

\begin{abstract}
To improve diagnostic options for pneumococcal pneumonia, an ELISA system was developed that can detect $\leqslant 6 \mathrm{ng} / \mathrm{ml}$ capsular polysaccharide in serum. The test was limited to 39 serotypes causing $>95 \%$ of pneumococcal infections. In clinical evaluation the test identified 14 of 15 cases (missing one serotype not included). No false-positive reaction occurred. However, the duration and level of antigenemia were variable $(\geqslant 500-2.5 \mathrm{ng} / \mathrm{ml})$ and seemed not to depend solely on the severity of infection. Therefore, the question of whether the extent of antigenemia was determined by a serotype-dependent variation in the elimination rates of polysaccharides was investigated. Clearance rates for 12 serotypes varied in rabbits and rats by a factor of $>250$. This remarkable variability appeared to affect the extent of clinical antigenemia. Thus, only very sensitive systems can detect circulating antigen from rapidly cleared polysaccharide serotypes. Furthermore, the question arises whether slow polysaccharide clearance contributes to the virulence of some pneumococcal serotypes.
\end{abstract}

Streptococcus pneumoniae still is one of the most important respiratory pathogens. Despite extensive research [1-8], diagnosis of nonbacteremic pneumococcal pneumonia remains an enigma $[9,10]$. It is estimated that nonbacteremic cases account for $70 \%-80 \%$ of pneumonias caused by S. pneumoniae. Results of sputum cultures or of sputum inoculation into mice are hampered mainly by a lack of specificity [2, 9-11], particularly in populations with a high incidence of chronic obstructive lung disease in which bronchial colonization with $S$. pneumoniae is common [12]. Furthermore, an appreciable number of patients with pneumonia do not produce sputum at the time they need antibiotic therapy $[7,9]$. Percutaneous lung aspiration has an impressive diagnostic yield with an acceptable complication rate when done by an experienced practitioner but has not gained wide popularity for fear of serious complications $[3,10,13]$.

Detection of pneumococcal antigens in body fluids for the diagnosis of pneumococcal pneumonia has a tradition of $>70$ years [14]. The method for detection of specific capsular polysaccharide (SCP) in blood and urine by counterimmunoelectrophoresis has been well characterized $[1,4]$ and is used in many centers despite its limited and serotype-dependent sensitivity $[1,7,9]$. The more convenient agglutination tests have provided a somewhat higher sensitivity in serum but not urine $[5,7,8]$. By using ELISA techniques and commercial sera,

Received 9 August 1990; revised 26 November 1990.

Presented in part: 28th Interscience Conference on Antimicrobial Agents and Chemotherapy, Los Angeles, October 1988 (abstract 463).

Grant support: Swiss National Science Foundation (3.875.0.85)

Reprints or correspondence: Dr. A. Schaffner, Laboratory for Clinical Mycology, Room AA23/28, Department of Medicine, University Hospital, CH-8091 Zurich, Switzerland.

The Journal of Infectious Diseases 1991;163:1094-1102 (C) 1991 by The University of Chicago. All rights reserved. $0022-1899 / 91 / 6305-0024 \$ 01.00$ the test sensitivity can be significantly improved for many but not all important SCP types [7, 15, 16]. In a small study, 12 of 17 cases of bacteremic pneumococcal pneumonia were detected by an ELISA technique based on IgG prepared from serum containing antibodies to all 84 known capsular polysaccharide types (Omniserum; Statens Serum Institute, Copenhagen), but for some SCP types this test system was relatively blind $[7,16]$.

Commercial sera are not specifically raised to react uniformly in ELISA systems but to produce uniform quellung reactions [17]. Avidity of an antibody is essential in ELISA systems [18] but might not be particularly important in the quellung reaction. Therefore, it seems possible that IgG raised specifically for use in an ELISA would make detection thresholds for different SCP types more similar. A test system reacting uniformly with all included SCP types would also permit estimation of blood levels of SCPs and provide insight into clearance rates of SCP types from blood, which might differ among the 84 pneumococcal serotypes.

Here we report an ELISA system developed with polyclonal rabbit IgG raised in our laboratory to ELISA specifications. The characterization and clinical evaluation of this ELISA system confirmed that by this tactic, uniformly low detection thresholds can be obtained for all included SCP types. For practical reasons we limited our test system to 39 serotypes that are responsible for $>95 \%$ of invasive pneumococcal infections $[17,19]$. We were, however, intrigued that serum levels of SCPs varied by a factor of $>150$ and that serum levels did not reflect the severity of pneumococcal infection. Thus, we systematically studied elimination of 12 different SCP types from blood in rats and rabbits.

\section{Methods}

Animals. Male New Zealand white rabbits (1.5-2.2 kg; (Madoring, Fullingsdorf, Switzerland) were kept singly under standard con- 
ditions. Female outbred, specific pathogen-free Wistar rats (Institute for Laboratory Animal Breeding, University of Zurich) were housed in groups of two to three and offered pelleted food and acidified water ad libitum.

Rabbit antibody. Rabbit antibody was raised in five pools, taking advantage of known cross-reactivities among SCP types [17], against the following pneumococcal strains (Danish nomenclature; Statens Serum Institute): pool A: 1, 2, 4, 5, 18F, 18A, 18B, 18C; pool B: 3, 6A, 6B, 8, 19A, 19F, 22F, 31; pool C: 7F, 7C, 13, 20, 24, 34, 35B; pool D: 9N, 9V, 10F, 10A, 11A, 11B, 17F, 23F; and pool E: 12F, 12A, 15A, 15B, 14, 16, 21, 33F. S. pneumoniae grown in serum-free Todd-Hewitt broth (BBL Microbiology Systems, Cockeysville, MD) to late-log phase was formol-fixed, washed, and stored as described by Lund and Henrichsen [17]. Male New Zealand white rabbits $(1.5-2 \mathrm{~kg}$ ) were immunized intravenously by three weekly injections of $1 \mathrm{ml}$ of the appropriate pool of formol-fixed bacteria adjusted to a density equivalent to $75 \%$ light transmission at $600 \mathrm{~nm}$.

Initially the bacterial pools were made by adding the same amount of each serotype (light transmission) to vaccines. Four weeks into immunization the pools were empirically adjusted for each rabbit by adjusting the proportion of a given serotype according to the antibody activity determined by ELISA every 2-3 weeks. Immunization was continued over 4-6 months before exsanguination. Antisera to single serotypes and serogroups, Omniserum, and anti-pool A serum for comparison with our sera were purchased from Statens Serum Institute. IgG was prepared by two consecutive $\mathrm{Na}_{2} \mathrm{SO}_{4}$ precipitations, followed by elution of IgG from a DEAE-Sephacel column [20]. IgG was coupled to horseradish peroxidase (RZ 3.6; Sigma, St. Louis) according to the periodate method of Wilson and Nakane [21].

Polysaccharide antigen was either obtained as mixture of 23 polysaccharides (Pneumovax-23; gift of Merck Sharp \& Dohme, West Point, PA) or as individual purified polysaccharides (ATCC, Rockville, $M D$ ) or prepared by threefold cold ethanol precipitation from deoxycholate-lysed pneumococci as described [17].

ELISA systems. Antibody titers in rabbits were measured in a whole-cell sandwich ELISA with washed, formol-fixed suspensions of pneumococci in V-bottomed microtiter plates. For the washing steps, plates with pneumococci were centrifuged at $1500 \mathrm{~g}$, and the supernatant was aspirated through a 21-gauge needle. Rabbit IgG was detected with a commercial anti-rabbit IgG-peroxidase conjugate (DAKO, Glostrup, Denmark). After production of the first anti-SCP IgG-peroxidase conjugates, titers were also followed by measuring SCP binding of serially diluted sera bound to microtiter plates. Antigen was detected and quantified by an antigen capture ELISA in which the solid phase was formed by ELISA microtiter plates (Immunlon type I; Dynatech, Alexandria, VA) coated overnight at $4^{\circ} \mathrm{C}$ with serotype- or serogroup-specific IgG in $0.05 M \mathrm{Na}_{2} \mathrm{CO}_{3}$, $\mathrm{pH} 9.5$. For capture of antigen, specimens were serially diluted in PBS and incubated at room temperature for $90 \mathrm{~min}$, followed by three washes and addition of the appropriate peroxidase-anti-pool antibody conjugate for $90 \mathrm{~min}$ more. Peroxidase was quantified by measuring peroxydation of $o$-phenylenediamine dihydrochloride (Sigma) in the presence of $\mathrm{H}_{2} \mathrm{O}_{2}$ by measuring the absorbance at $492 \mathrm{~nm}$ in an automated ELISA reader (Dynatech) after addition of $\mathrm{H}_{2} \mathrm{SO}_{4}$ to stop the reaction. Antibody dilutions for coating microtiter plates and of peroxidase conjugates were optimized by checkerboard titration. Antigen concentrations were computed from linear regression curves obtained from serial dilutions of pure antigen in the appropriate diluent.
Study population. The ward teams of our medical department, a tertiary referral center, were invited during a 12 -month period to submit blood, urine, sputum, and cerebrospinal and pleural fluid from patients in whom pneumococcal infection was considered at admission. Only patients from whom a blood specimen was submitted within $36 \mathrm{~h}$ of admission were evaluated. A total of $144 \mathrm{epi}-$ sodes was studied in 143 patients. In 98 episodes, pneumococcal infection was considered possible by the ward teams; those later excluded on clinical grounds served as controls. Blood samples from 46 additional patients hospitalized for elective cardiovascular surgery without signs of infection or chronic bronchitis were included as additional controls. Pneumonia was defined as a disease presenting with an unequivocal radiologic lung infiltrate and systemic signs of inflammation without evidence for an alternate diagnosis (e.g., pulmonary infarction).

Preparation of clinical specimens for antigen detection. Serum or plasma was diluted 1:5 and 1:10 with PBS before heating for 10 min in boiling water to denature proteins and centrifugation at 3000 $g$ for $10 \mathrm{~min}$. Cerebrospinal and pleural fluid was equally heated. Urine was concentrated 20 -fold by cold ethanol precipitation and resuspension of the precipitate in PBS [17].

Kinetic studies in animals. For kinetic studies in rabbits, polysaccharide in a dose of 250 or $500 \mu \mathrm{g}$ suspended in 5 or $10 \mathrm{ml}$ of isotonic saline was injected into an ear vein, after bleeding to determine background activity. At indicated times, $5 \mathrm{ml}$ of blood was obtained from the ear arteries. In rats, blood antigen levels were studied after intraperitoneal injection of antigen or log-phase pneumococcal cultures resuspended in PBS to a light transmission of $75 \%$ $(600 \mathrm{~nm})$. Colony-forming units of the challenge dose were enumerated after culture of serial dilutions on blood agar plates in candle jars. Blood from rats was obtained from the retroorbital venous plexus of anesthetized animals. Within 20 min after challenge with live bacteria, rats were administered $10^{6}$ units of procaine penicillin intramuscularly to halt infection.

Statistical analysis. Simple linear regression curves were constructed by using the Inplot Graphpad program (Graphpad, San Diego) on an IBM AT computer. Mean values were compared by unpaired $t$ test. For intertest comparison of absorbance readings, ELISA readings were adjusted for each microtiter plate by subtracting the mean background and multiplying the readings from samples with a factor derived from interior positive standards included in all microtiter plates (corrected optical density [OD]). The formula was (OD of the test sample - OD of the test background) [(mean OD of all standards - mean OD of the background)/(OD of the test standard - OD of the test background)]. In kinetic studies, serum half-lives of antigens were computed from linear regression curves (Graphpad). Volumes of distribution were computed by dividing the injected dose of polysaccharide by the serum concentration at the time of injection computed from the elimination curve. Clearance rates were computed by the formula: clearance $=(0.7 \cdot \mathrm{vol}-$ ume of distribution)/T1/2.

\section{Results}

Production of antisera and characterization of ELISA. By strictly adhering to the procedures outlined by Lund and Henrichsen [17] for the production of vaccines and immunization of rabbits, anti-SCP pool antisera were obtained without difficulty. Starting 4 weeks after the beginning of immuniza- 
Table 1. Antibody titer of a commercial anti-pool A serum raised to give uniform quellung reactions and an anti-pool A serum raised to give uniform ELISA titers: relation of antibody titer by ELISA to the sensitivity of the antigen-detection ELISA.

\begin{tabular}{cccccc} 
& \multicolumn{2}{c}{ Commercial serum } & & \multicolumn{2}{c}{ Elisa serum } \\
\cline { 2 - 3 } \cline { 5 - 6 } Serotype & $\begin{array}{c}\text { Antibody } \\
\text { titer }\end{array}$ & $\begin{array}{c}\text { Detection } \\
\text { threshold } \\
(\mathrm{ng} / \mathrm{ml})\end{array}$ & & $\begin{array}{c}\text { Antibody } \\
\text { titer }\end{array}$ & $\begin{array}{c}\text { Detection } \\
\text { threshold } \\
(\mathrm{ng} / \mathrm{ml})\end{array}$ \\
\hline 1 & $1: 64,000$ & 0.63 & & $1: 256,000$ & 0.16 \\
2 & $1: 32,000$ & 1.33 & & $1: 256,000$ & 0.16 \\
4 & $1: 32,000$ & 2.5 & & $1: 256,000$ & 0.63 \\
5 & $1: 32,000$ & 0.63 & & $1: 64,000$ & 0.63 \\
$18 \mathrm{C}$ & $1: 8000$ & 2.5 & & $1: 128,000$ & 0.63 \\
\hline
\end{tabular}

NOTE. Commercial anti-pool A rabbit serum was compared with a serum specifically raised to obtain uniformly high titers by whole-cell ELISA. The detection threshold for purified commercial antigen (ATCC) was measured in buffer by capture sandwich ELISA optimized for each antigen by checkerboard titration.

tion, the composition of the seven or eight serotypes making up a pool vaccine was adjusted individually for each rabbit by augmenting the fraction of formol-fixed bacteria from the serotype to which the antibody response was inferior compared with the average for the other serotypes in the same pool or reducing the fraction of a serotype if antibody to one serotype prevailed in the pool. Immunotolerance was never a problem after increasing the vaccine dose. After 4-6 months of continuous immunization, sera deemed adequate were obtained for all five antigen pools.

Antibody titers and detection threshold for five purified antigens are shown for anti-pool $\mathrm{A}$ serum in table 1. So that we could compare our sera raised to ELISA specification with a commercial serum raised to produce a uniform quellung reaction (Statens Serum Institute), we included the same number and types of pneumococci in our anti-pool A serum as in theirs. While both sera showed comparable variability in reactivity with individual serotypes, the serum raised to ELISA specification had a $>5$ times higher mean antibody titer by ELISA and detected on average a 3.4 times lower antigen concentration, regardless of concentrations of capture antibodies and conjugates established for each individual antigen, serum, and conjugate. These observations indicated that it was advantageous to follow the antibody response during serum production by ELISA, which in contrast to the quellung reaction also permitted an objective quantitation of antibody.

When we tested the quality of our ELISA system with a representative panel of the most commonly isolated pneumococcal serotypes, the ELISA detected relatively uniformly low concentrations of polysaccharides in human serum (mean \pm $\mathrm{SD}, 2.3 \pm 1.24 \mathrm{ng} / \mathrm{ml}$; table 2 ), even though the samples were diluted 1:5 in PBS for heat inactivation of nonspecific reactions between rabbit serum and clinical specimens.

Clinical evaluation. Sera from 143 patients (144 episodes) obtained within $36 \mathrm{~h}$ of admission were studied. Antigen was detected in sera from 14 of 15 patients with blood cul-
Table 2. Detection threshold of ELISA for specific capsular polysaccharides in normal human serum.

\begin{tabular}{lc}
\hline Serotype & Threshold $(\mathrm{ng} / \mathrm{ml})$ \\
\hline 1 & 1.56 \\
2 & 1.56 \\
3 & 1.56 \\
4 & 3.12 \\
5 & 3.12 \\
$6 \mathrm{~A}$ & 1.56 \\
$7 \mathrm{~F}$ & 1.56 \\
$8 \mathrm{~N}$ & 0.78 \\
$9 \mathrm{~N}$ & 1.56 \\
$10 \mathrm{~A}$ & 6.25 \\
$12 \mathrm{~F}$ & 3.12 \\
14 & 1.56 \\
$15 \mathrm{~B}$ & 3.12 \\
$18 \mathrm{C}$ & 3.12 \\
$19 \mathrm{~F}$ & 1.56 \\
$22 \mathrm{~F}$ & 1.56 \\
$23 \mathrm{~F}$ & 3.12 \\
\hline
\end{tabular}

NOTE. Normal human serum was spiked with $100 \mathrm{ng} / \mathrm{ml}$ of individual purified polysaccharides (ATCC) and serially diluted $1: 2$ with normal human serum. Threshold concentration is original polysaccharide concentration before $1: 5$ dilution and heat inactivation detectable in spiked normal human serum. Cutoff was mean of all negative samples +2 SD.

Table 3. Study population.

\begin{tabular}{|c|c|c|}
\hline Group & No. patients & $\begin{array}{l}\text { No. positive } \\
\text { by ELISA }\end{array}$ \\
\hline I, definite* pneumococcal infection & 15 & 14 \\
\hline II, pneumococcal disease excluded $\dagger$ & 28 & 0 \\
\hline III, cardiovascular control group & 46 & 0 \\
\hline IV, pneumonia of unknown cause $t$ & 55 & 11 \\
\hline
\end{tabular}

* Pneumonia, 8; meningitis, 4; bacteremia without definite pulmonary infiltrales, 3; required isolation of Streptococcus pneumoniae from blood.

$\dagger$ Fourteen noninfectious conditions (malignancies, pulmonary infarction, cardiac failure, chronic obstructive lung disease), 14 pneumonias caused by alternate pathogens diagnosed by culture or serology $(Q$ fever, legionellosis, subphrenic empyema with pleural effusion, tuberculosis [2], Streptococcus pyogenes pneumonia [2], Staphylococcus aureus pneumonia [2], chronic obstructive lung disease with viral infection [5]).

Pulmonary infiltrate on chest radiograph, fever, laboratory findings of inflammation but no etiologic diagnosis (three sets of blood cullures, negative serologic workup)

ture-proven pneumococcal infection. One blood isolate, which reacted with Omniserum but with none of our pool sera or the full set of 39 commercial anti-serotype or -serogroup sera, was not included in our five seropools, explaining the one negative test. This episode of pneumococcal bacteremia occurred in an individual who had been vaccinated several months previously with the 23 -valent pneumococcal vaccine after posttraumatic splenectomy, a setting possibly favoring infection with a less common serotype. No serum was positive in the 28 control patients in which pneumococcal infection was considered possible at entry but excluded on clinical grounds. False-positive results also were not a problem in 46 control patients with cardiovascular disease without signs of infection or inflammation (table 3 ).

The ELISA also was evaluated in other body fluids submit- 
Table 4. Detection of polysaccharide in body fluids other than serum.

\begin{tabular}{lllc}
\hline & & \multicolumn{2}{c}{$\begin{array}{c}\text { Cerebrospinal } \\
\text { or pleural }\end{array}$} \\
Group & Urine & Sputum & fluid \\
\cline { 4 - 5 } I, definite pneumococcal infection & $10 / 10$ & $5 / 5$ & $7 / 7$ \\
II, pneumococcal disease excluded & $1 / 13$ & $5 / 8$ & $0 / 9$ \\
IV, pneumonia of unknown cause & & & \\
A: positive serum ELISA & $5 / 8$ & $4 / 5$ & NA \\
B: negative serum ELISA & $6 / 31$ & $8 / 16$ & $0 / 2$ \\
\hline
\end{tabular}

NOTE. Data are number positive/number tested. Positive urine and cerebrospinal and pleural fluid samples always reacted in the same seropool as the serum sample. In contrast, sputum samples reacted frequently in additional seropools in groups I and IVA. All group II and most group IVB patients with positive sputum reactions and all patients with positive urine reactions had a history of chronic productive bronchitis. The urine reaction was always concordant with one of the frequently multiple reactions of the five seropools with sputum. NA: no specimen available.

Table 5. Serotyping of antigen in body fluids by ELISA.

\begin{tabular}{lclc}
$\begin{array}{l}\text { Reactive } \\
\text { pool } \\
\text { (ELISA) }\end{array}$ & $\begin{array}{c}\text { Reactive } \\
\text { type or } \\
\text { group }\end{array}$ & $\begin{array}{c}\text { Source } \\
\text { (body fluid) }\end{array}$ & $\begin{array}{c}\text { Serotype or } \\
\text { serogroup of } \\
\text { isolate }\end{array}$ \\
\hline A & 2 & S, U & 2 \\
A & 4 & S, PLF, U & 4 \\
C & 7 & S, PLF & 7 \\
C & 35 & S, CSF & 35 \\
D & 9 & S, CSF & 9 \\
D & 9 & S, U & 9 \\
D & 23 & S & 23 \\
\hline
\end{tabular}

NOTE. A set of anti-serotype or -serogroup sera corresponding to the reactive pool was used as capture antibody to bind type- or group-specific antigen followed by the detection step with anti-pool conjugate. S, serum; PLF, pleural fluid; U, 20-times-concentrated urine; CSF, cerebrospinal fluid.

ted together with the sera (table 4). These studies confirmed $[11,12]$ that the airways in patients with chronic obstructive lung disease are frequently colonized by one or multiple pneumococcal serotypes, so that little gain can be expected from an increase in sensitivity of a pneumococcal detection system. Furthermore, in some patients with mucopurulent chronic bronchitis, antigen of the same pool type as in sputum was detected in 20-fold-concentrated urine, indicating that by lowering the detection threshold for antigen in urine, antigenuria associated with bronchial colonization could produce false-positive results.

By using commercial serotype- and serogroup-specific antisera as capture antibody, antigen could be typed correctly in body fluids by ELISA from the seven patients from whom an isolate was available for typing by the quellung reaction (table 5).

Extent of antigenemia. The specifications required for a test system to be adequately sensitive to diagnose pneumococcal infection by detecting SCP in serum is governed by the level of clinical antigenemia. When we looked at the ELISA readings from 14 positive serum samples from bacteremic patients, it was not a problem to discriminate their positive readings from that of 28 patients in which pneumococcal in-

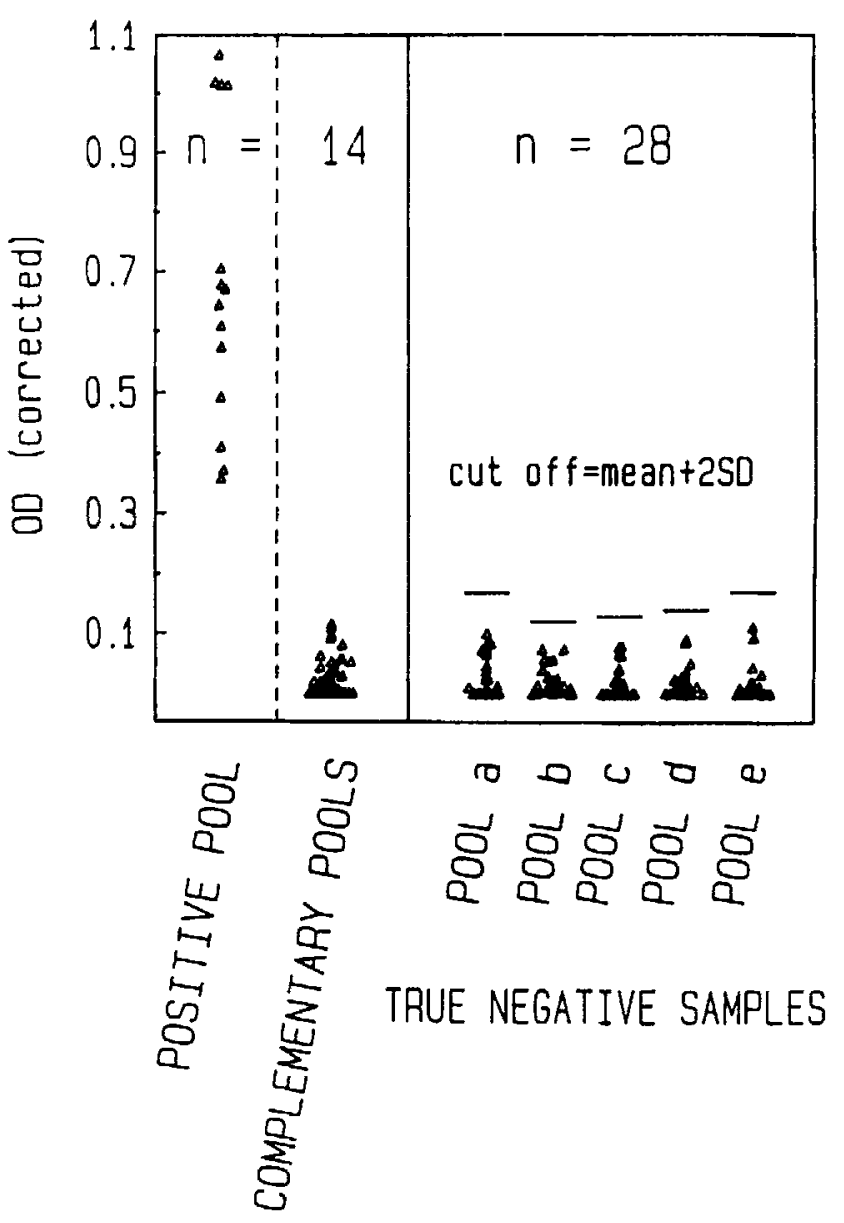

Figure 1. ELISA readings from 14 patients with blood culturepositive pneumococcal infection (positive pool and complementary negative pools). There was no cross-reaction between positive seropool and four complementary pools. A cutoff 2 SD above mean value of negative controls, in whom pneumococcal infection was excluded (table 3, group II), and negative complementary pools of proven cases frankly discriminates positive and negative test results. Optical density (OD) read at $492 \mathrm{~nm}$ was corrected for intertest variations by internal standards $O D$.

fection was excluded. Furthermore, antigen did not cross-react with complementary pools in positive sera (figure 1). However, when we looked at the nonbacteremic individuals with pneumococcal pneumonia, diagnosed on the basis of a positive ELISA test, we observed a discrete distribution of ELISA readings, ranging from clear-cut positive values to readings barely above the cutoff point, fixed $2 \mathrm{SD}$ above the mean value of the negative readings from bacteremic patients (complementary pools) and those in which pneumococcal infection was excluded (figures 1 and 2). Because our ELISA system reacted uniformly with all included antigens, this observation indicated that the level of antigenemia was disturbingly inconsistent. This was confirmed by computing the antigen level from linear regression curves plotted for each seropool. This analysis showed, not unexpectedly, that bacteremic pneumococcal infections were usually associated with higher levels of 


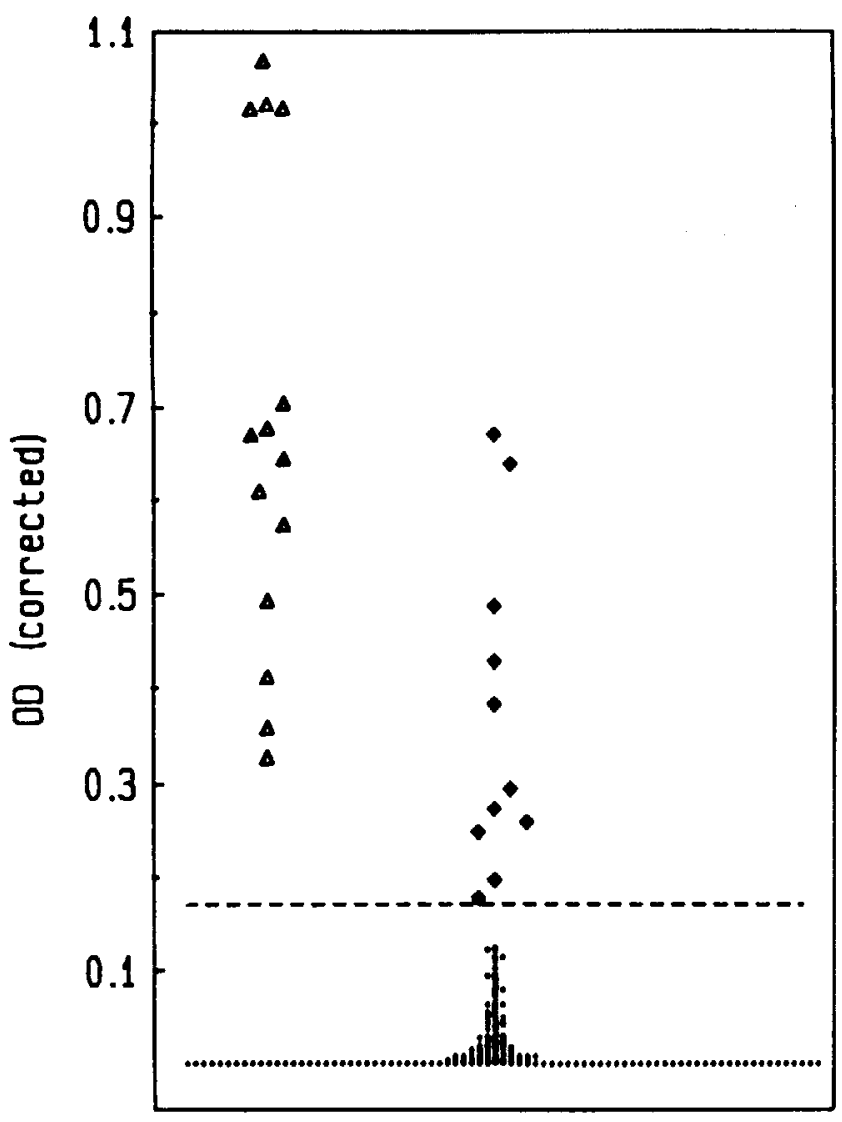

Figure 2. ELISA readings from 55 patients with blood cultureand serologically negative pneumonia, 11 with antigen detected in serum by ELISA $(\diamond)$ and 44 with no antigen detected $(\bullet)$. Note that these ELISA readings, in contrast to those from bacteremic pneumococcal infections $(\Delta)$, show discrete distribution without clearcut bimodal distribution of positives and negatives. Dashed horizontal line corresponds to highest cutoff for negative readings, which was for pool A (see figure 1).

antigenemia than were nonbacteremic cases (table 6). But the variability of antigen levels by a factor of $>150$ at the time of bacteremia was unexpected. Furthermore, in many patients antigen levels (table 6) were below the detection threshold reported for antigen detection systems based on commercial sera $[5,7,8,15,16]$.

These observations on the variability of antigenemia pointed to unknown factors that affected the level of antigenemia. Further insight was gained into such possible factors by comparing the evolution of antigenemia in two patients with bacteremic pneumococcal pneumonia, both adequately treated with penicillin $G$, to which the isolates were highly sensitive. One patient with pneumonia and sepsis caused by $S$. pneumoniae serotype $7 \mathrm{~F}$ was initially in septic shock but cleared antigenemia within the first 3 days. The second patient with comparable pulmonary infiltrates caused by $S$. pneumoniae serotype 2 was unable to clear antigenemia over the full length of his hospital stay (figure 3). These observations indicated that the elimination rate of SCP could differ dramatically and
Table 6. Antigen concentrations detected in serum by ELISA.

\begin{tabular}{|c|c|c|c|c|c|}
\hline \multicolumn{3}{|c|}{$\begin{array}{c}\text { Group I: } \\
\text { Bacteremic pneumococcal } \\
\text { pneumonia }\end{array}$} & \multicolumn{3}{|c|}{$\begin{array}{c}\text { Group IVA: } \\
\text { Nonbacteremic } \\
\text { pneumococcal pneumonia* }\end{array}$} \\
\hline Pool & $\begin{array}{l}\text { Type or } \\
\text { group }\end{array}$ & $\begin{array}{c}\text { Level } \\
\text { (ng/ml) }\end{array}$ & Pool & $\begin{array}{l}\text { Type or } \\
\text { group }\end{array}$ & $\begin{array}{l}\text { Level } \\
\text { (ng/ml) }\end{array}$ \\
\hline A & 2 & 360.0 & A & & 10.0 \\
\hline A & $1^{\dagger}$ & 39.0 & B & & 8.0 \\
\hline A & 4 & 10.0 & B & & 3.5 \\
\hline B & $22 \dagger$ & 500.0 & B & $8^{\dagger}$ & 2.5 \\
\hline B & & 81.0 & $\mathrm{C}$ & & 15.5 \\
\hline B & & 6.0 & $\mathrm{C}$ & $7^{\dagger}$ & 11.5 \\
\hline B & & 4.5 & C & & 5.0 \\
\hline C & 7 & 242.0 & $\mathrm{C}$ & & 3.5 \\
\hline $\mathrm{C}$ & 35 & 51.0 & $\mathrm{C}$ & & 3.0 \\
\hline D & 9 & 18.0 & $\mathrm{D}$ & & 15.5 \\
\hline D & & 16.0 & $\mathrm{D}$ & $9^{\dagger}$ & 13.5 \\
\hline D & 9 & 10.0 & & & \\
\hline D & 23 & 7.5 & & & \\
\hline D & & 3.5 & & & \\
\hline Mean $\pm \mathrm{SD}$ & & $96.3 \pm 156^{\ddagger}$ & & & $8.3 \pm 5^{\ddagger}$ \\
\hline
\end{tabular}

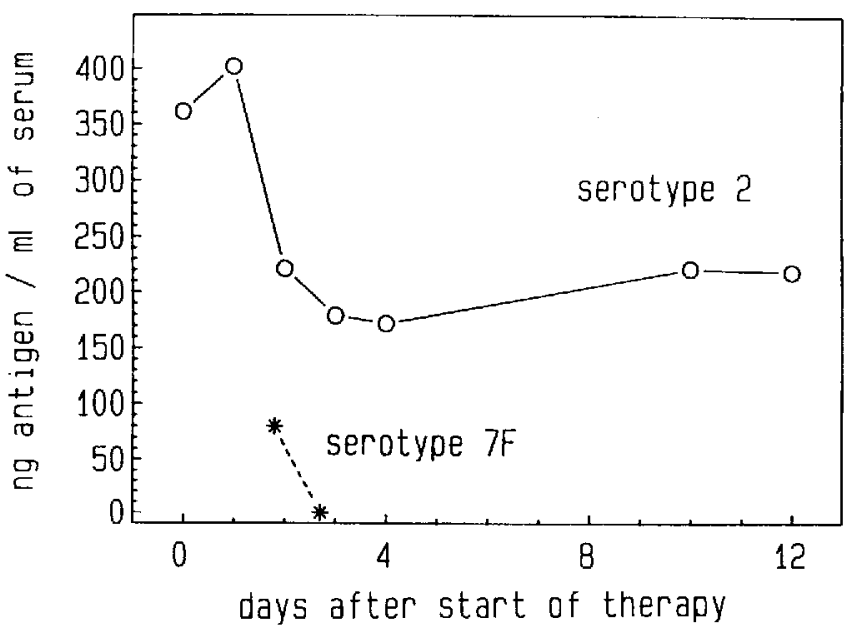

Figure 3. Course of antigenemia in a patient with serotype 2 and a patient with serotype $7 \mathrm{~F}$ pneumonia of similar severity.

presumably affected blood antigen levels. We therefore turned to experimental studies on the variability of SCP clearance from blood.

Kinetic studies of antigenemia in animals. In accordance with our clinical observations, rats rapidly eliminated immunoreactive SCP from serotype 7F but not from serotype 2 from their blood (figure 4).

Next we compared systematically the elimination rates of 12 SCP types in rabbits. These studies showed that the serum half-life of immunologically detected SCPs could vary by a factor of $>70$, ranging for the 12 examined serotypes from 24 min to $>29$ h (figure 5). In addition to serum half-life of 


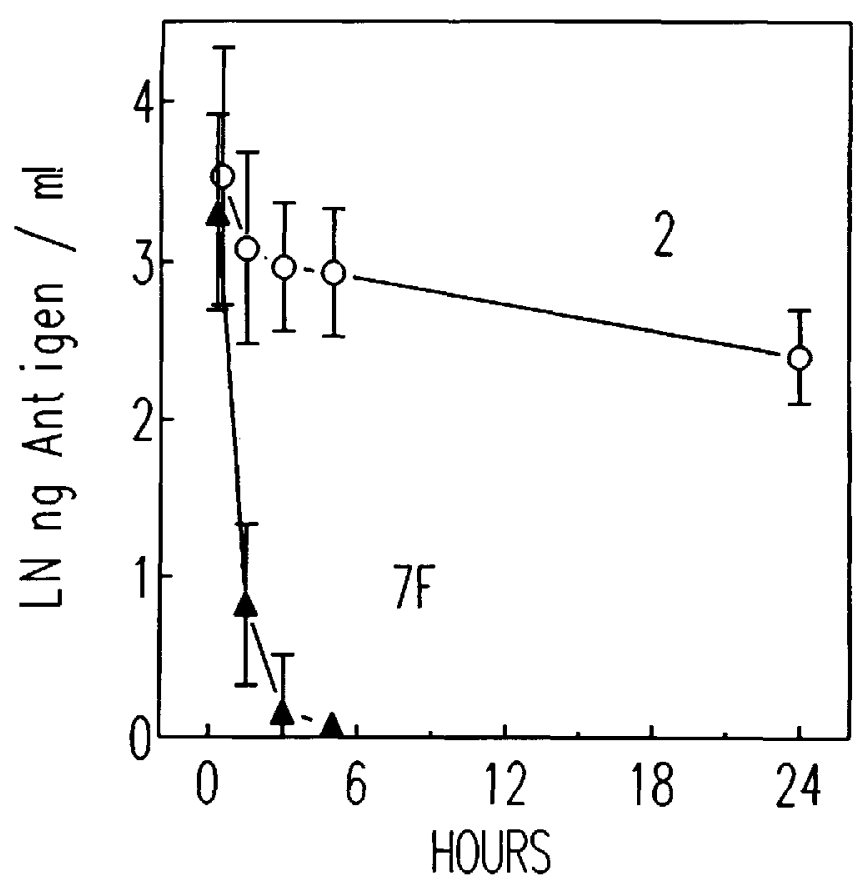

Figure 4. Elimination of specific capsular polysaccharides 2 and 7F from blood. Groups of five rats were injected with $1 \mathrm{mg}$ (serotype 2) or $2 \mathrm{mg}$ (serotype 7F) of a crude preparation of capsular polysaccharide into the peritoneal cavity, and antigen levels were followed by ELISA for $24 \mathrm{~h}$. Data are mean \pm SD. $L N=\log _{e}$.
SCPs, their volume of distribution and therefore their clearance varied appreciably (table 7). The mechanisms behind the variability in SCP serum half-lives and their distribution volumes appeared not to be uniformly the same because no correlation was seen between the two kinetic parameters (table 7). According to the variations in serum half-life, the period during which antigen could be detected in serum varied significantly (table 7) and correlated well with the serum halflife but not with the volume of distribution.

Next we explored the possibility that polysaccharides would preferentially associate with formed blood elements. We compared the antigen concentration measured in blood plasma with that of sedimented blood cells for 11 of the 12 selected serotypes (all but 10A). The ratio of the polysaccharide concentration in the plasma to that in the cell sediment was $>1$ for all antigens tested except for $11 \mathrm{~A}$ and 20 , which were preferentially associated with formed blood elements. When we attempted to correlate the volume of distribution or the elimination rate from blood to the relative distribution of polysaccharide between plasma and the formed blood elements we found no significant correlation between these parameters (not shown). Nevertheless, this study indicated that for a few selected serotypes sedimented blood cells were a more promising source for diagnostic antigen detection than serum or plasma.
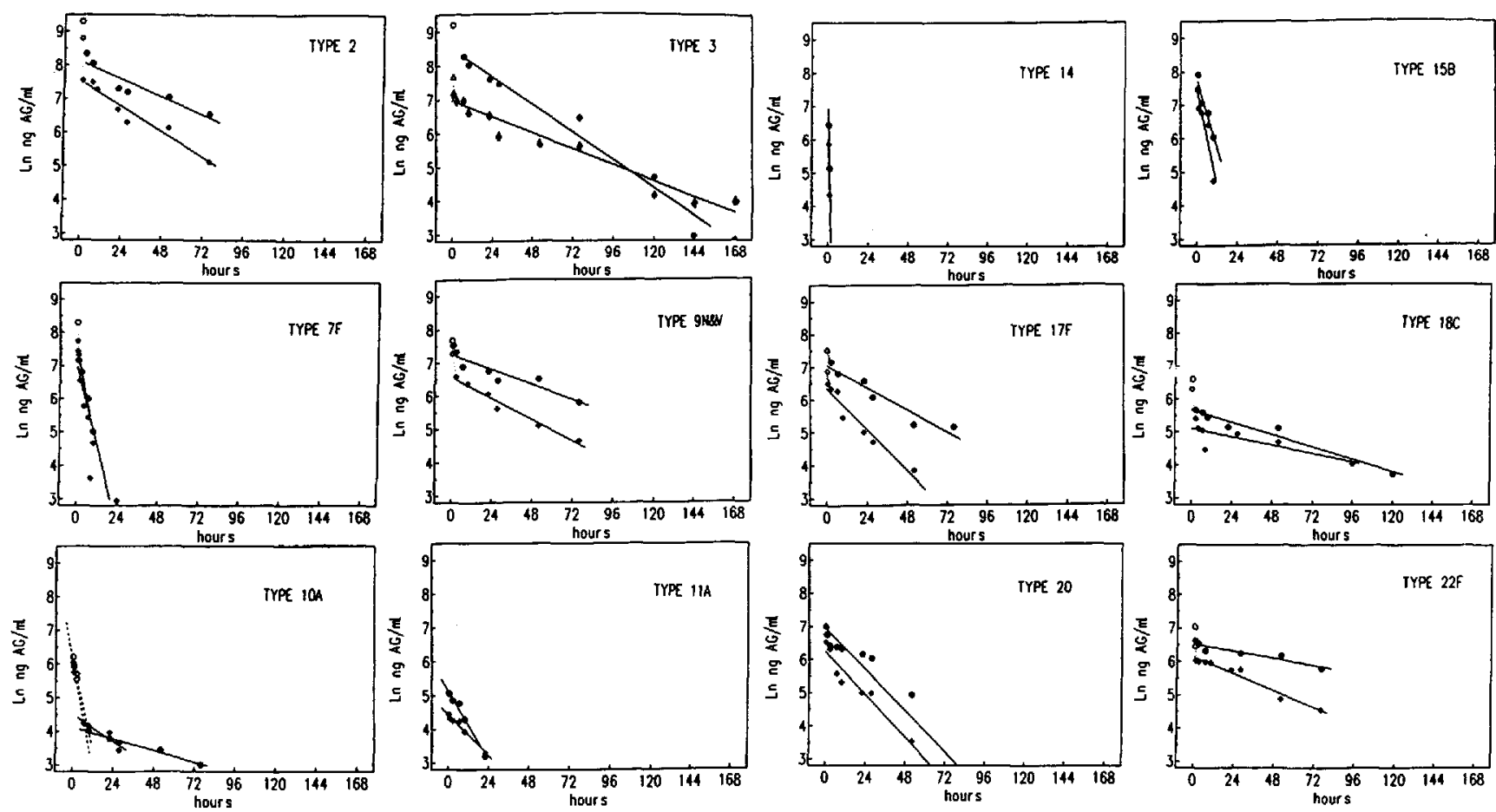

Figure 5. Kinetic of the disappearance of pneumococcal polysaccharides from serum of a rabbit injected intravenously with $500 \mu \mathrm{g}$ (circles) or $250 \mu \mathrm{g}$ (diamonds) of polysaccharide. In some instances the phase of initial distribution was not completed 15 min after injection of the antigens (open symbols). Linear regression was computed from antigen levels after completion of initial distribution (solid symbols). Type 10A did not fit a model of a first-order elimination kinetic but showed two distinct phases, with a first half-time of $2.7 \mathrm{~h}$ followed by a slower terminal phase with a half-time of $39 \mathrm{~h} . \mathrm{LN}=\log _{\mathrm{e}}$. 
Table 7. Volume of distribution, serum half-life, clearance, and time period with detectable antigenemia in rabbits after intravenous injection of pneumococcal capsular polysaccharides.

\begin{tabular}{lcccc}
\hline & $\begin{array}{c}\text { Volume of } \\
\text { distribution } \\
\text { (ml/kg) }\end{array}$ & $\begin{array}{c}\text { Serum } \\
\text { half-life } \\
\text { Serotype }\end{array}$ & $\begin{array}{c}\text { Clearance } \\
\text { (ml/min/kg) }\end{array}$ & $\begin{array}{c}\text { Duration of } \\
\text { anti- } \\
\text { genemia }\end{array}$ \\
\hline 2 & $47.3 \pm 8$ & $20.0 \pm 8.0$ & 1.6 & 83 \\
3 & $88.8 \pm 14$ & $27.5 \pm 10.0$ & 2.3 & 144 \\
$7 \mathrm{~F}$ & $90.3 \pm 14$ & $3.1 \pm 1.0$ & 20.4 & 13 \\
$9 \mathrm{~N} \& \mathrm{~V}$ & $245.2 \pm 15$ & $24.0 \pm 9.0$ & 7.2 & 83 \\
$10 \mathrm{~A}$ & $1026.0 \pm 103$ & $19.3 \pm 1.0$ & 37.2 & 40 \\
$11 \AA$ & $1231.0 \pm 38$ & $7.1 \pm 5.0$ & 123.1 & 8 \\
14 & $214.5 \pm 22$ & $0.4 \pm 0.1$ & 375.4 & 2 \\
$15 \mathrm{~B}$ & $62.4 \pm 15$ & $2.6 \pm 1.0$ & 16.8 & 9 \\
$17 \mathrm{~F}$ & $129.5 \pm 30$ & $23.7 \pm 12.0$ & 3.8 & 75 \\
$18 \mathrm{C}$ & $479.0 \pm 82$ & $29.1 \pm 8.0$ & 11.5 & 99 \\
20 & $192.0 \pm 3$ & $13.7 \pm 1.0$ & 9.8 & 67 \\
$22 \mathrm{~F}$ & $265.9 \pm 54$ & $26.1 \pm 8.0$ & 7.1 & 83 \\
\hline
\end{tabular}

NOTE. Serum half-life was computed from linear regression after completion of the initial distribution of injected antigen. Clearance was computed from the apparent volume of distribution of the injected antigen and the serum half-life. Data are mean or mean \pm SD from three animals. Antigen (Pneumovax-23) was injected intravenously in a dose of $250 \mu \mathrm{g}$ in two animals and $500 \mu \mathrm{g}$ in one animal for 7 days for each serotype. Data were pooled because elimination rates appeared not to depend on antigen load at these doses.

We next ascertained that the examined antigens were stable in heparinized rabbit blood and serum by demonstrating that the concentration of all 12 antigens was not altered by incubation in blood or serum over $48 \mathrm{~h}$ at $37^{\circ} \mathrm{C}$.

Because antibody to SCP might affect its elimination from the blood, we studied whether antibody was responsible for the observed variations between serum half-lives of SCPs. We examined whether natural antibody was responsible for the disparities, because adaptive humoral immunity developing in response to injected SCP could not be of importance in view of the stable elimination rates attained within a few hours after challenge with antigen. When we looked at the potential of serum from rats or rabbits obtained before challenge to capture polysaccharide in our sandwich ELISA system, we could not detect any SCP binding to the solid phase mediated by the sera. Similarly, formol-fixed pneumococci did not bind detectable amounts of rabbit or rat serum immunoglobulin from the animals under study.

We anticipated that SCP types with a short serum half-life would not easily build up high blood SCP levels during the course of an infection. To confirm this we turned again to rats; we used a model of infection with our initially studied pair of SCP-types with variable elimination rates to study the kinetics of antigenemia after infection with live bacteria (figure 6 ). These studies confirmed that the rapidly cleared SCP type 7F does not build up high antigen levels in the course of infection, while infection with $S$. pneumoniae serotype 2 results in consistently high SCP levels, which even increase after the beginning of penicillin treatment. This experiment also confirmed that the discrepant elimination rates of SCP of sero-

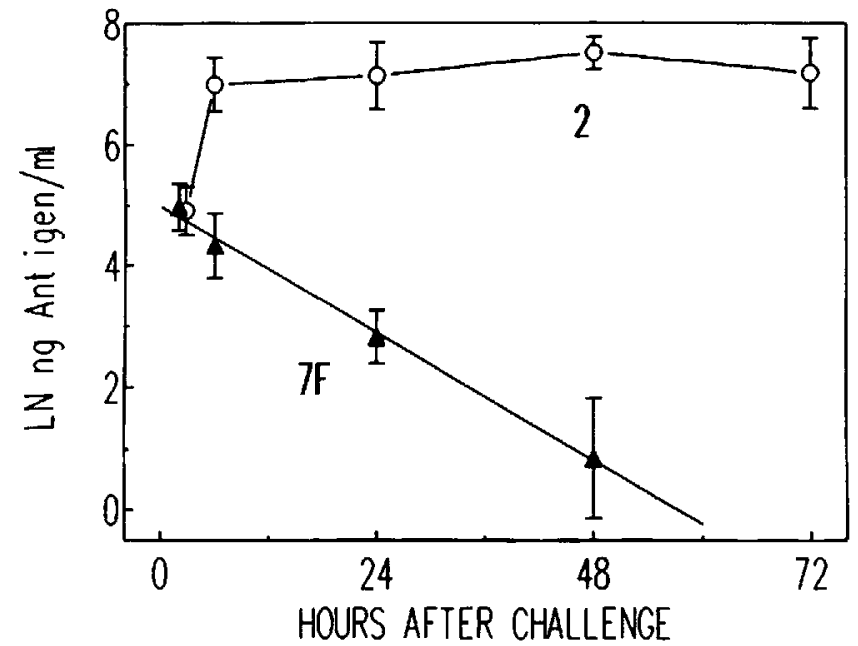

Figure 6. Kinetics of antigenemia of rats challenged with live pneumococci serotypes 2 and $7 \mathrm{~F}$ into the peritoneal cavity 20 min before administration of $10^{6}$ units of procaine penicillin intramuscularly. Data are mean \pm SD from four or five rats per time point. Challenge dose was $4.8 \times 10^{8} \mathrm{cfu}$. $\mathrm{LN}=\log _{\mathrm{e}}$.

type 2 and 7F did not result from an artifact stemming from in vitro antigen preparation because the difference between the two elimination rates was comparable in the experiments with purified antigen and live bacteria (Figures 4 and 6).

\section{Discussion}

These studies show that by raising antisera to pneumococcal polysaccharides specifically for use in ELISA, it is possible to obtain a clinically useful and highly and uniformly sensitive tool for the diagnosis of invasive pneumococcal infection. Our ELISA test correctly identified at admission all 14 bacteremic cases caused by serotypes included in the test. The only infection missed was caused by a pneumococcal serotype not contained in our pools of 39 serotypes. The test also detected antigenemia in 11 of 55 patients with blood culture-negative pneumonia that remained without etiologic diagnosis after a complete workup, increasing the number of diagnosed pneumococcal pneumonias from 8 to 19 among 63 prospectively studied pneumonia patients (table 3 ).

The diagnostic yield of our ELISA compares favorably with previous studies evaluating the increased sensitivity of ELISA over that of counterimmunoelectrophoresis or agglutination techniques for diagnosing pneumococcal pneumonia $[6-9,15$, 16]. In the best-documented study [7], in which an ELISA based on Omniserum was evaluated, 9 of 17 bacteremic pneumococcal infections were diagnosed on the initial sample and 12 of 17 with subsequent blood samples. In view of the limited sensitivity of this Omniserum-based ELISA, which ranged from 200 to $3000 \mathrm{ng} / \mathrm{ml}$ of serum for single SCP types, it is not surprising that this ELISA missed five bacteremic cases and was not useful for the diagnosis of nonbacteremic pneu- 
monias, in which we found antigen levels $10-100$ times below the detection limit of the Omniserum-based test (table 6). ELISA systems developed with commercial anti-pool sera have been found to be more sensitive than Omniserum systems [16], but even such an ELISA system was relatively blind for certain common serotypes, resulting in false-negative results even in culturally positive cerebrospinal fluid samples [16].

In any event, these studies indicate that detection of SCP in blood by an ELISA able reliably to detect antigen levels as low as $1-6 \mathrm{ng} / \mathrm{ml}$ of serum can significantly contribute to the diagnosis of pneumococcal infection, even in settings such as nonbacteremic pneumococcal pneumonia. Furthermore, the ability to serotype antigen in clinical specimens would allow further insight into the epidemiology of nonbacteremic pneumococcal pneumonia or even rapid prediction of the risk for penicillin resistance that is associated with certain serotypes [22]. In contrast to detection by blood tests, the increased sensitivity for detection of SCP in concentrated urine specimens or sputum was offset by the many positive reactions in sputum and urine of patients with chronic obstructive lung disease accompanied by chronic bronchitis and pneumococcal colonization (table 4).

Our study documents an impressive variability in the blood levels of SCP antigen, by a factor of $>150$; this variability could not be related to the severity of the infection alone. Bacteremic patients had higher antigen levels, as shown previously by others [23], but even during bacteremia the variability remained impressive (table 6). On one hand the inconsistent antigen levels, with concentrations as low as $3-10 \mathrm{ng} / \mathrm{ml}$ in bacteremic cases and levels at the detection limit of our assay in nonbacteremic cases, documented that to be clinically reliable, a test for detection of antigenemia must have a detection threshold of as little as $1-2 \mathrm{ng} / \mathrm{ml}$ or even less. On the other hand, the variability of antigenemia indicated that apart from the severity of infection and the presence of bacteremia, additional, hitherto-unknown factors determine the extent of antigenemia. Because we observed an impressive difference in the elimination rate of SCP in two patients with comparably severe infections caused by disparate SCP serotypes, the question occurred whether the elimination of SCP was serotype dependent.

Animal studies of experimental antigenemia confirmed that disposal of immunoreactive capsular pneumococcal polysaccharides varies significantly among different serotypes. Serum half-life, the volume of distribution, and the clearance rates of the 12 examined SCP types differed by a factor of $>70,25$, and 250 , respectively, explaining why some SCP types were detectable for $>7$ days in experimental antigenemia while others were eliminated from blood within hours (table 7).

The observation that the elimination of SCP types 2 and $7 \mathrm{~F}$ from the blood of rabbits and rats was comparable in both species and, above all, corresponded to the clinical observations with these two SCP types that originally prompted these studies (figures 3-6) indicates that the variability of SCP clearance rates is, at least in principle, not species dependent. These findings have obvious implications for the clinical use of SCP detection in body fluids for diagnosis of pneumococcal infection. It is conceivable that SCP types rapidly eliminated from blood are more difficult to detect in circulation, building up lower serum levels that last for shorter time periods. Accordingly, the experimental observations are in agreement with our clinical findings that the extent of antigenemia did not correlate with the severity of pneumococcal infections and that the antigen blood levels measured in a population of patients with serious pneumococcal infection showed a disturbing variability, with values from as high as several hundred $\mathrm{ng} / \mathrm{ml}$ to barely measurable levels no longer clearly distinct from antigen-free samples in a sensitive ELISA system (figure 2). Furthermore, the significant variations in the clearance of polysaccharide from blood and the important differences between the volume of distribution for the studied polysaccharides make it plausible that excretion of antigen in the urine, another body fluid used for antigen detection [7, 24], must also be quite variable.

Our observations also raise the question whether prolonged persistence of some SCP types contributes to virulence. The injection of homologous pneumococcal capsular polysaccharides impressively enhances the susceptibility of mice to pneumococci $[25,26]$. Circulating polysaccharide antagonizes homologous humoral anti-pneumococcal defenses by neutralizing antibody [25-28], and it appears logical that SCP types reaching higher levels and circulating for longer time periods have more impact on humoral defenses by neutralizing antibody than do polysaccharides that fail to circulate for extended periods. In this context, it is interesting that Coonrod and Drennan [23] noted that pneumococci with low serotype numbers, which might be more virulent than highnumbered serotypes, cause higher SCP blood levels than do high-numbered serotypes and that high levels of antigenemia are associated with delayed appearance of measurable antibody. Finally, failure to eliminate polysaccharide from circulation might contribute to the phenomenon of immunologic paralysis $[29,30]$, reported clinically with polysaccharide $18 \mathrm{C}$ [31], which had the longest circulation time among the 12 SCP types studies in our experiments.

\section{References}

1. Coonrod JD, Rytel MW. Detection of type specific pneumococcal antigens by counterimmunoelectrophoresis. Etiologic diagnosis of pneumococcal pneumonia. J Lab Clin Med 1973;81:778-86.

2. Leach RP, Coonrod JD. Detection of pneumococcal antigens in the sputum in pneumococcal pneumonia. Am Rev Respir Dis 1977;116: 847-51.

3. Davidson M, Tempest B, Palmer DL. Bacteriologic diagnosis of acute pneumonia: comparison of sputum, transtracheal aspirates, and lung aspirates. JAMA 1976;235:158-63.

4. Perlino A. Laboratory diagnosis of pneumonia due to Streptococcus pneumoniae. J Infect Dis 1984;150:139-44. 
5. Martin SJ, Hoganson DA, Thomass ET. Detection of Streptococcus pneumoniae and Haemophilus influenzae type b antigens in acute nonbacteremic pneumonia. J Clin Microbiol 1987;25:248-50.

6. Holmberg H, Krook A. Comparison of enzyme-linked immunosorbent assay with coagglutination and latex agglutination for rapid diagnosis of pneumococcal pneumonia by detecting antigen in sputa. Eur J Clin Microbiol Infect Dis 1986;5:282-6.

7. Lenthe-Eboa $S$, Brighouse G, Auckenthaler R, et al. Comparison of immunological methods for diagnosis of pneumococcal pneumonia in biological fluids. Eur J Clin Microbiol Infect Dis 1987;6:28-34.

8. Ortquist A, Jonsson I, Kalin M, Krook A. Comparison of three methods for detection of pneumococcal antigen in sputum of patients with community-acquired pneumonia. Eur J Clin Microbiol Infect Dis 1989;8:956-61.

9. Rytel MW, Preheim LC. Antigen detection in the diagnosis and in the prognostic assessment of bacterial pneumonias. Diagn Microbiol Infect Dis 1986;4:\$35-46.

10. Palmer DL, Jones CC. Diagnosis of pneumococcal pneumonia. Semin Respir Infect 1988;3:131-9.

11. Barrett-Connor $\mathrm{E}$. The nonvalue of sputum culture in the diagnosis of pneumococcal pneumonia. Am Rev Respir Dis 1971;103:845-8.

12. Haas, H, Morris JF, Samson S, Kilbourn JP, Kim JP. Bacterial flora of the respiratory tract in chronic bronchitis: comparison of transtracheal, fiber-bronchoscopic and oropharyngeal sampling methods. Am Rev Respir Dis 1977;116:41-7.

13. Barnes DJ, Naraqi S, Igo JD. The role of percutaneous lung aspiration in the bacteriological diagnosis of pneumonia in adults. Aust $\mathrm{N} \mathrm{Z}$ J Med 1988;18:754-7.

14. Dochez AR, Avery OT. The elaboration of specific soluble substance by pneumococcus during growth. J Exp Med 1917;26:477-92.

15. Harding SA, Sheld WM, McGowan MD, Sande MA. Enzyme-linked immunosorbent assay for detection of Streptococcus pneumoniae antigen. J Clin Microbiol 1979;10:339-42.

16. Da Costa Castro JM, Deschamps F, Benbachir M, Henrichsen J, Volle PJ, Guinet RMF. Highly sensitive biotin-avidin sandwich ELISA for the rapid detection of pneumococcal capsular polysaccharide antigens. J Immunol Methods 1987;104:265-70.

17. Lund E, Henrichsen J. Laboratory diagnosis, serology and epidemiology of streptococcus pneumoniac. Methods Microbiol 1978;12:241-62.
18. Roitt I. Essential immunology. Oxford: Blackwell Scientific Publications, 1980.

19. Austrian R. Some observations on the pneumococcus and on the current status of pneumococcal disease and its prevention. Rev Infect Dis 1981;3:S1-17.

20. Johnstone A, Thorpe R. Immunochemistry in practice. Oxford: Blackwell Scientific Publications, 1982.

21. Wilson MB, Nakane PK. Recent development in the periodate method of conjugating horseradish peroxidase (HRPO) to antibodies. In: Knapp W, Holulas $\mathrm{K}$, Wick G, eds. Immunofluorescence and related staining techniques. New York: Elsevier-North Holland Biomedical Press, 1978:215-24.

22. Austrian R. Pneumococcal polysaccharide vaccines. Rev Infect Dis 1989;11:S598-612.

23. Coonrod JD, Drennan DP. Pneumococcal pneumonia: capsular polysaccharide antigenemia and antibody responses. Ann Intern Med 1976;84:254-60.

24. Coonrod JD. Urine as an antigen reservoir for diagnosis of infectious diseases. Am J Med 1983;75:85-92.

25. Felton LD, Bailey GH. Biological significance of the soluble specific substances of pneumococci. J Infect Dis 1926;38:131-44.

26. Felton LD, Kaufmann G, Prescott B, Ottinger B. Studies on the mechanism of the immunologic paralysis induced in mice by pneumococcal polysaccharides. J Immunol 1955;74:17-26.

27. Rosenow EC. Human pneumococcal opsonin and the anti-opsonic substance in virulent pneumococci. J Infect Dis 1907;4:285-96.

28. Cole R. The neutralization of antipneumococcus immune bodies by infected exudates and sera. J Exp Med 1918;27:453-575.

29. Stark OK. Studies on pneumococcal polysaccharide. II. Mechanism involved in production of "immunologic paralysis" by type I pneumococcal polysaccharide. J Immunol 1955;74:130-3.

30. Howard JG, Christie GH, Jakob MJ, Elson J. Studies on immunological paralysis. III. Recirculation and antibody-neutralizing activity of ${ }^{14} \mathrm{C}$ labeled type III pneumococcal polysaccharide in paralyzed mice. Clin Exp Immunol 1970;7:583-96.

31. Pichiero ME. Immunological paralysis to pneumococcal polysaccharide in man. Lancet 1985;2:468-71. 